\title{
Safeguarded Use of the \\ Implicit Restarted Lanczos Technique for Solving Nonlinear Structural Eigensystems
}

M.R. Abdel-Aziz

June, 1993

TR93-27 



\title{
SAFEGUARDED USE OF THE IMPLICIT RESTARTED LANCZOS TECHNIQUE FOR SOLVING NONLINEAR STRUCTURAL EIGENSYSTEMS
}

\author{
Mohammedi R. Abdel-Aziz, Research Associate \\ Department of Computational and Applied Mathematics, \\ Rice University, Houston, TX 77251.
}

July 2, 1993 



\section{SUMMARY}

This paper presents a new algorithm for evaluating the eigenvalues and their corresponding eigenvectors for large scale nonlinear eigensystems in structural dynamics. The algorithm is based on solving a sequence of algebraic eigenproblems and updating the parameter, $\lambda$. The Implicitly Restarted Lanczos method has been determined to be well suited for solving the linear eigenproblems that are arised in this context. A zero-finder approach that uses rational interpolation to approximate the generalized eigenvalues has been developed to update $\lambda$.

The methodology of the new algorithm developed here is designed to evaluate a subset of the parametrized nonlinear eigencurves at a specific values of $\lambda$. Numerical experiments show that the new eigensolution technique is superior to the pre-existing approaches for the large scale problems and comptitive for the small size ones. The main emphasis of this contribution is the derivation and analysis of this scheme for eigensystems that are based on the frequency dependent shape functions.

\section{INTRODUCTION}

Large scale eigenvalue problems are of great importance in many physical areas. These very large problems arise through the discretization of a differential operator to approximate some of its spectral properties. However, there are many additional sources for these problems. Saad gave a number of examples in ${ }^{17}$. One of the most important sources for these large problems is the vibration analyses of structures which is concerned with accurately predicting the free vibration modes and frequencies (eigenvectors and eigenvalues) of the vibrating structure. The vibration analysis process involves two main parts :

- The finite element model of the physical system determines the number of equations of motion needed to exactly define the behavior of the vibrating system. The finite element models can be distinguished by the shape function utilized by the model.

- The numerical technique computs the vibration modes and frequencies of the system 
from the resulting eigenproblem.

The eigenproblems may be linear or nonlinear depending on the choice of the shape functions approximated or exact. This choice plays a crucial role in both solution accuracy and efficiency. The linear eigenproblem results from the conventional finite element model. This model uses polynomial shape functions to approximate the solution of the differential equation that governs the system. This problem takes the form

$$
[\mathbf{K}-\lambda \mathbf{M}] \mathbf{x}=\mathbf{0}
$$

where, $\mathbf{M}$ and $\mathbf{K}$ are the system mass and stiffness matrices which are symmetric positive definite, respectively, $\lambda$ is the eigenvalue (the square of the natural frequency, $\omega$ ) and $x$ is its corresponding eigenvector. These problems may be nonsymmetric or symmetric. The Arnoldi process ${ }^{1}$ is an appropriate technique for finding a few of eigenvalues and their corresponding eigenvectors of a large nonsymmetric square matrix . For large symmetric eigenproblems, we use a variant of Lanczos algorithm ${ }^{7}$. There are also many methods that depend on similarity transformations to solve the linear eigenvalue problems, but these methods are not useful for large problems ${ }^{25,28}$.

The eigenproblems associated with the exact displacement model ${ }^{13}$ and the mixed formulation model ${ }^{21}$ take the forms

$$
\begin{aligned}
{[\mathbf{K}(\lambda)-\lambda \mathbf{M}(\lambda)] \mathbf{x} } & =\mathbf{0} \text { and } \\
{[\mathbf{K}-\lambda \mathbf{M}(\lambda)] \mathbf{x} } & =\mathbf{0} .
\end{aligned}
$$

The matrices $\mathbf{K}(\lambda)$ and $\mathbf{M}(\lambda)$ are the system stiffness and mass matrices derived from the potential and the kinetic energy expressions, respectively. These matrices are symmetric and their entries are functions of $\lambda$

Newton's method may be used to solve these problems, either by dealing with the problem as a system of nonlinear equations or by expanding the dynamic stiffness matrix via Taylor series and then repeatedly solve the matrix pencil obtained from the first two terms of Taylor series expansion ${ }^{15,32}$. Some drawbacks of these procedures are that the derivative 
of the dynamic stiffness matrix may not exist or it may be hard to evaluate. Newton's techniques are locally $q$-quadratic in the neighborhood of the solution, but not globally convergent in general. Thus, they may need another method for computing a good initial guess and they must be safeguarded. An intensive research has been developed in this field by Swannell ${ }^{27}$, Richards and Leung ${ }^{14}$, Hallauer and Liu ${ }^{4}$ and Simpson ${ }^{18}$. Williams and Wittrick have studied nonlinear symmetric eigenproblems in ${ }^{30,31}$. Algorithms presented there depend on determinant evaluation and the bisection method for updating the parameter $\lambda$. Of course the bisection technique is safe and easy to implement but it is relatively slow to converge. $\operatorname{In}^{29}$ Williams and Kennedy give an improved zero-finding algorithm by combining parabolic interpolation with bisection method but it is slow for large problems.

This study presents and discusses a new iterative algorithm for evaluating a subset of the smallest eigenvalues $\lambda$ and their corresponding eigenvectors $x$ for the large scale, dependent, sparse and symmetric eigenproblems which is defined in formula (1.2). This formula can be written as :

$$
\mathbf{M}(\lambda) \mathbf{x}=\mu(\lambda) \mathbf{K} \mathbf{x}, \quad \mu(\lambda)=\frac{1}{\lambda}
$$

The features of this formulation are the matrix $K$ is factorized once for all the iterations and the transformation $\frac{1}{\lambda}$ gives large well separated eigenvalues. The generalized eigenvalues, $\mu(\lambda)$ are monotone increasing at specific values of the parameter $\lambda$ on an interval containing the solution. This was observed in ${ }^{23}$ and proved for special cases in ${ }^{20}$.

This new procedure does not require any derivatives or determinant evaluation. It involves two main parts: solving linear eigenvalue problems by using a suitable eigen-solver and updating the approximated eigenvalue by using a zero-finding technique.

This paper is organized as follows: In section 2, we describe Lanczos and Implicit Restarted Lanczos (IRL) methods which are used to solve the symmetric linear eigenproblem that arise. We give a brief description of employing these methods to solve the nonlinear eigenvalue problem (1.3). Section 3 presents two zero-finders that are based on linear and rational interpolation. In section 4, we developed a safeguarded rational zero- 
finder which is a combination of the zero-finders that are based upon the interpolation. Section 5 is devoted to establish our algorithm that is based on the idea linearize, solve and then update. In section 6 , we introduce the comparison with some competitive techniques and some numerical experiments. Concluding remarks are given in the last section.

\section{LANCZOS ALGORITHM}

The Lanczos algorithm may be viewed as a projection technique on a Krylov subspace. It begins with the specification of the starting vector $\mathbf{v}_{1} \in R^{n}$, and a real symmetric matrix $\mathbf{A} \in R^{n \times n}$. In $k$-steps, Lanczos process produces the decomposition of $\mathbf{A}$ into the form

$$
\mathbf{A V}=\mathbf{V T}+\mathbf{f e}_{k}^{t}
$$

where $\mathbf{V} \in R^{n \times k}, \mathbf{V V}^{t}=\mathbf{I}_{k}, \mathbf{T} \in R^{k \times k}$ is tridiagonal, $\mathbf{f} \in R^{n}$ with $\mathbf{V}^{t} \mathbf{f}=0$ and $\mathbf{e}_{k}$ is the $k$-th co-ordinate basis vector. The approximated eigenvalues and eigenvectors are available through this factorization. If $(\theta, y)$ is the eigenpair of $\mathbf{T}$, then the pair $(\theta, \mathbf{x})$ is an approximated eigenpair of $A$ where, $\mathbf{x}=\mathbf{V y}$ and the error of the approximation is given by

$$
\|\mathbf{A x}-\theta \mathbf{x}\|=\|\mathbf{f}\|\left|\mathbf{e}_{k}^{t} \mathbf{y}\right|
$$

The vector $\mathbf{f}$ is called the residual vector, for $\mathbf{f}=\mathbf{0}$ or $\mathbf{e}_{k}^{t} \mathbf{y}=0$ will imply that the columns of $\mathbf{V}$ form an orthonormal basis for an invariant subspace of $\mathbf{A}$ and that the eigenvalues of $\mathbf{T}$ are exact eigenvalues of $\mathbf{A}$. The major practical difference between this process and the non-hermitian methods is that we only need to save three vectors, at least if we do not restore to any form of reorthogonalization. In addition, the largest and smallest eigenvalues will emerge well before the tridiagonalization is completed. Because of these features, Lanczos method is especially attractive for applications when a few of the largest or smallest eigenvalues are of interest. Unfortunately, the Lanczos process has some numerical difficulties resulting from the loss of orthogonality among the Lanczos vectors, the appearance of spurious eigenvalues in the spectrum of $\mathbf{T}$, the error due to finite precision 
arithmetic, and the undetermined storage requirement. These drawbacks have been the subject of considerable research over the last two decades ${ }^{3,16,2}$.

For sake of completeness of this study, suppose Lanczos process is applied until $k$-steps then it can continue further $p$-steps by using

$$
\text { function }[\mathbf{T}, \mathbf{V}, \mathbf{f}]=\operatorname{Lanczos}(\mathbf{A}, \mathbf{T}, \mathbf{V}, \mathbf{f}, k, p)
$$

For more details about Lanczos process and the main steps of this function (2.1), we refer the reader to $^{8}$.

\subsection{The IRL Method}

The computational difficulties which mentioned above stem from the fact that the residual $\|\mathbf{f}\|=0$, if and only if the columns of $\mathbf{V}$ span an invariant subspace of $\mathbf{A}$. When $\mathbf{V}$ "nearly" spans such a subspace, $\|\mathbf{f}\|$ will be small. Typically, in this situation, a loss of significant digits will take place. These issues can be controlled by limiting the number of steps in the Lanczos process at a certain value $k$ and then treating the residual vector as a function of the initial Lanczos vector. The starting vector is then updated through a polynomial filter scheme which is designed to force convergence of the residual to zero ${ }^{22}$. The Lanczos function (2.1) can be used to describe a complete iteration of IRL method as follows:

\section{Algorithm 2.1}

function $[\mathbf{T}, \mathbf{V}, \mathbf{f}]=\operatorname{IRL}(\mathbf{A}, \mathrm{k}, \mathrm{p}$, tol $)$.

(1) Initialize $\mathbf{V}(:, \mathbf{1})=\mathbf{v}_{1} ; \mathbf{T} \leftarrow\left(\mathbf{v}_{\mathbf{l}}^{t} \mathbf{A} \mathbf{v}_{1}\right) ; \mathbf{f} \leftarrow \mathbf{A} \mathbf{v}_{\mathbf{1}}-\mathbf{v}_{\mathbf{1}} \mathbf{T}$;

(2) $[\mathbf{T}, \mathbf{V}, \mathbf{f}] \leftarrow \operatorname{Lanczos}(\mathbf{A}, \mathbf{T}, \mathbf{V}, \mathbf{f}, 1, \mathrm{k})$

(3) For $m=1,2, \ldots$ until convergence

(a) if $(\|\mathbf{f}\|<t o l)$ then stop;

(b) $[\mathbf{T}, \mathbf{V}, \mathbf{f}] \leftarrow \operatorname{Lanczos}(\mathbf{A}, \mathbf{T}, \mathbf{V}, \mathbf{f}, \mathrm{k}, \mathrm{p})$; 
(c) $u=\operatorname{Shifts}(\mathbf{T}, \mathrm{p})$;

(d) $Q \leftarrow \mathbf{I}_{k+p}$;

(e) for $j=1,2, \ldots, p$

(e.1) $\mathbf{T} \leftarrow \mathbf{Q}_{j}^{t} \mathbf{T} \mathbf{Q}_{j} ;\left(\right.$ Bulge-Chase with shift $\left.\gamma_{j}=u(j)\right)$

(e.2) $\mathbf{Q} \leftarrow \mathrm{QQ}_{j}$;

(4) $\mathbf{v} \leftarrow(\mathbf{V Q}) \mathbf{e}_{k+1} ; \mathrm{V} \leftarrow(\mathbf{V Q})\left(\begin{array}{c}\mathbf{I}_{k} \\ \mathbf{0}\end{array}\right)$;

(5) $\mathbf{f} \leftarrow\left(\mathbf{v} \beta_{k}+\mathbf{f} \sigma_{k}\right)$; where $\beta_{k}=\mathbf{e}_{k+1}^{t} \mathbf{T} \mathbf{e}_{k}, \sigma_{k}=\mathbf{e}_{k+p}^{t} \mathbf{Q \mathbf { e } _ { k }}$;

The Bulge Chase at step (3.e.1) is defined implicitly as usual so that $\mathbf{T}-\gamma_{j} \mathbf{I}=\mathbf{Q}_{j} \mathbf{R}_{j}$. There is no need for applying the implicit double shift since all the eigenvalues are real. For details and alternative strategies for choosing shifts see ${ }^{22}$.

\subsection{The nonlinear eigenproblem solution using IRL method}

The IRL technique has been used with a considerable success to solve different large scale algebraic eigenvalue problems up to dimension $10^{4}$ degrees-of-freedom on a variety of machines ${ }^{22}$. Results from ${ }^{23}$ show the mixed model results are nearly exact when they compared with the results from ${ }^{19}$.

These advantages of IRL method and the structure of the nonlinear eigenvalue problems associated with the mixed finite element model (1.2) indicate that the use of IRL technique to solve the linear eigenproblems will give an accurate and efficient results. In the formulation (1.2), Lanczos factorization becomes

$$
\mathbf{M}(\lambda) \mathbf{V}=\mathbf{K V T}(\lambda)+\mathbf{f e}_{k}^{t}
$$

with the columns of $\mathbf{V}$ being $\mathbf{K}$-orthogonal, and $\mathbf{T}(\lambda)$ is tridiagonal. Let $\mu_{\mathbf{I}}(\lambda)$ be the first largest generalized eigenvalue of $\mathbf{T}(\lambda)$ and let $\mathbf{y}_{1}$ be the corresponding eigenvector. The value $\mu_{1}(\lambda)$ is the approximation of the first largest generalized eigenvalue of problem (1.3) 
at specific value of $\lambda$. This is typically, found very rapidly with the IRL iteration [12]. A solution to the nonlinear problem (1.3) is obtained when we have solved the scalar equation

$$
\mu_{1}(\lambda)=\frac{1}{\lambda} \text { under the condition }\|\mathbf{f}\|\left|\mathbf{e}_{\mathrm{k}}^{\mathrm{t}} \mathrm{y}_{1}\right|<\epsilon \mu_{1}(\lambda)
$$

A variety of standard and specialized numerical techniques (such as bisection technique, Newton-Raphson's method and secant approach) may be applied to this scalar problem.

The only way that the symmetric matrix $\mathbf{A}$ is involved in the computation is during the step that required matrix vector products. Thus, the product Av needs to be formed most economically by a procedure, which takes full advantage from the sparsity and the symmetry of $\mathbf{K}$ and $\mathbf{M}(\lambda)$. It does not required an explicit formulation of $\mathbf{A}$. The product $\mathbf{A}(\lambda) \mathbf{v}_{k}=\mathbf{L}^{-1} \mathbf{M}(\lambda) \mathbf{L}^{-t} \mathbf{v}_{k}$ can be computed without additional cost by factorizing the matrix $\mathbf{K}$ only one time. The factorization was subsequently used repeatedly to compute this matrix vector product at each $\lambda$. Assume that the vectors $\mathbf{z}$ and $\mathbf{u}$ are temporary $n \times 1$ vector arrays and the matrix $\mathbf{K}$ has been factored to $\mathbf{L L}^{\mathbf{t}}$ using Cholesky factorization. The following algorithm is used for computing the product $\mathbf{A}(\lambda) \mathbf{v}_{k}$.

\section{Algorithm 2.2}

function $[\mathbf{w}]=$ product $\left(\mathbf{L}, \mathbf{M}(\lambda), \mathbf{v}_{k}\right)$

(a) Solve : $\mathbf{L}^{t} \mathbf{u}=\mathbf{v}_{k}$ for $\mathbf{u}$ by a back substitution;

(b) Form : $\mathbf{z}=\mathbf{M}(\lambda) \mathrm{u}$

(c) Solve : $\mathbf{L w}=\mathrm{z}$ for $\mathrm{w}$ by a forward substitution.

Instead of solving problem (1.3) the transformed problem

$$
\mathbf{A}(\lambda) \mathbf{x}=\mu(\lambda) \mathbf{x}
$$

will be solved. The problem becomes standard eigenvalue problem at specific values of $\lambda$ and $\mathbf{A}(\lambda)$ is still symmetric. The Lanczos function (2.1) is determined such that the step 
which requires matrix vector products is replaced by algorithm 2.2 , i.e $\mathbf{w}=\mathbf{A}(\lambda) \mathbf{v}_{k}$. The IRL technique (algorithm 2.1) becomes

$$
\text { function }[\mathbf{T}, \mathbf{V}, \mathbf{f}]=\operatorname{IRL}(\mathbf{A}, \mathbf{L}, \mathrm{k}, \mathrm{p}, \text { tol })
$$

with the same steps but it calls the Lanczos function (2.1) which calls algorithm 2.2.

The entries of the matrix $\mathbf{A}(\lambda)$ are ratios of transcendental functions. The generalized eigenvalues of the matrix $\mathbf{A}(\lambda)$ at specific values of $\lambda$ are the roots of a characteristic polynomial that has rational coefficients. The relationship between the roots and the coefficients of the polynomials lead us to approximate the generalized eigenvalues $\mu(\lambda)$, with rational functions. The IRL method (2.3) can be applied to evaluate a subset of the largest generalized eigenvalues $\mu(\lambda)$ for the linear eigenvalue subproblems that appear during the solution of problem (2.2). A scalar zero-finder based on rational interpolation can be applied for updating the eigenvalue $\lambda$. The solution of this problem, (2.2) is the intersection points between $\frac{1}{\lambda}$-curve and $\mu(\lambda)$-curves as shown in figure 1 .

\section{SOME ZERO-FINDING TECHNIQUES}

The root-finding approachs that are used for updating the parameter $\lambda$ in the symmetric nonlinear eigenvalue problems may be regarded as an outer iteration for finding the root of the equation $\mu(\lambda)=\frac{1}{\lambda}$. Once the value of $\lambda$ is updated the next evaluation of $\mu(\lambda)$ will use the first column of $\mathbf{V}$ to begin the new restarted Lanczos process. The latest eigenvalue, $\lambda$ from the zero-finder with the largest generalized eigenvalue from the eigen-solver will be used to initiate the next zero-finding step.

The interpolation methods to be discussed in this section are very useful for determining zeros of arbitrary real functions $\mu(\lambda)$. They have the advantage that the derivatives of $\mu(\lambda)$ need not be computed. The best methods available for zero-finding are the so-called safeguarded procedures. The idea is to combine a guaranteed, reliable method with a rapidly convergent method, to yield an algorithm that will converge at the rate of the safe guaranteed method if $\mu(\lambda)$ is well - behaved ${ }^{26}$. 


\subsection{Secant Method}

Secant technique has convergence rate $\frac{1}{2}(1+\sqrt{5})$, but it may take several function evaluations to find a root. In addition, it may not match a highly nonlinear function well because it is based on the linear interpolation. The following is a description for constructing the first required two points.

1. Initialize the eigenvalue $\lambda$ with $\lambda_{1}$;

2. Solve : $\mathbf{A}\left(\lambda_{1}\right) \mathbf{x}=\mu\left(\lambda_{1}\right) \mathbf{x}, \mu_{1} \leftarrow \max \mu\left(\lambda_{1}\right), \lambda_{2} \leftarrow \frac{1}{\mu_{1}}$

3. Solve : $\mathbf{A}\left(\lambda_{2}\right) \mathbf{x}=\mu_{\left(\lambda_{2}\right)} \mathbf{x}, \mu_{2} \leftarrow \max \mu\left(\lambda_{2}\right)$;

A straight line between $\left(\lambda_{1}, \mu_{1}\right)$ and $\left.\left(\lambda_{2}, \mu_{2}\right)\right)$ can be constructed. It intersects the curve $\frac{1}{\lambda}$ at the solution point. The point $\left(\lambda_{1}, \mu_{1}\right)$ is set to be $\left(\lambda_{2}, \mu_{2}\right)$ and the solution point is set to be $\lambda_{2}$. Again the matrix $\mathbf{A}\left(\lambda_{2}\right)$ is computed and a linear eigenproblem is solved. We continue to do these secant approximations using the most updated value of $\lambda$ until the stopping criterion is satisfied ${ }^{23}$.

\subsection{Rational Zero-finder}

In problem 2.2, the entries of the matrix $\mathbf{A}(\lambda)$ are composed of elementary transcendental functions approximated well by rational functions. These features are reflected in the generalized eigenvalues $\mu(\lambda)$ so that special techniques must be derived to ensure convergence to physically meaningful results. The well-known secant method gives remarkable results to the solution of equation $\mu(\lambda)=\frac{1}{\lambda}$. However, it still takes several iterations to find a root. Methods based upon the rational function interpolation have been used to great advantage in solving trust region problems ${ }^{10}$. The value $\mu(\lambda)$ is a root of a polynomial whose coefficients are rational functions of the matrix entries $A(\lambda)$. So, the approximation of the function $\mu(\lambda)$ can be written as :

$$
\mu(\lambda) \approx \frac{\alpha \lambda+\beta}{\gamma \lambda+1}
$$


which has the advantage of being linear when $\gamma$ equals zero. The constants $\alpha, \beta$, and $\gamma$ are determined by interpolating $\mu(\lambda)$ at three distinct points. The first two points can be found as in subsection 3.1. The goal is to find the third point without increasing the number of function evaluations (i.e without solving more linear eigenvalue problems). The function $\mu(\lambda)$ has a monotone increasing behavior, figure 1 . Thus, the third point can be found as follows :

Apply : the secant method for one step between $\left(\lambda_{1}, \mu_{1}\right)$ and $\left(\lambda_{2}, \mu_{2}\right)$ to find the secant point, $\lambda_{s}$.

Determine : $\quad \lambda_{3} \leftarrow \lambda_{2}, \mu_{3} \leftarrow \mu_{2}, \lambda_{2} \leftarrow \lambda_{s}$ and $\mu_{2} \leftarrow \mu_{1}+\epsilon\left(\epsilon=10^{-6}\right)$. Once the third point found, we solve

$$
\left(\begin{array}{ccc}
1 & \lambda_{1} & -\mu_{1} \lambda_{1} \\
1 & \lambda_{2} & -\mu_{2} \lambda_{2} \\
1 & \lambda_{3} & -\mu_{3} \lambda_{3}
\end{array}\right)\left(\begin{array}{l}
\beta \\
\alpha \\
\gamma
\end{array}\right)=\left(\begin{array}{l}
\mu_{1} \\
\mu_{2} \\
\mu_{3}
\end{array}\right)
$$

for $\alpha, \beta$, and $\gamma$. The larger root of the quadratic, $\alpha \lambda^{2}-(\gamma-\beta) \lambda-1=0$ is the updated value of $\lambda$. A new iteration starts by solving a new linear eigenvalue problem at $\lambda_{2}$ (the most updated value resulting from the previous iteration) to find $\mu_{2}=\max \mu\left(\lambda_{2}\right)$. Apply the secant method between $\left(\lambda_{2}, \mu_{2}\right)$ and $\left(\lambda_{3}, \mu_{3}\right)$ to find the secant point, $\lambda_{s}$. The new points are $\left(\lambda_{2}, \mu_{2}\right),\left(\lambda_{s}, \mu_{2}+\epsilon\right)$ and $\left(\lambda_{3}, \mu_{3}\right)$ which are set to be $\left(\lambda_{1}, \mu_{1}\right),\left(\lambda_{2}, \mu_{2}\right)$ and $\left(\lambda_{3}, \mu_{3}\right)$, respectively. We continue to do this process after solving the linear system and the quadratic equation until the stopping criterion is satisfied.

This strategy has some drawbacks that can lead to non-convergence. These problems are described as follows :

1. The matrix in the linear system (3.2) becomes

$$
\left(\begin{array}{ccc}
1 & \lambda_{1} & -\mu_{1} \lambda_{1} \\
0 & \lambda_{2}-\lambda_{1} & \mu_{1} \lambda_{1}-\mu_{2} \lambda_{2} \\
0 & 0 & \theta
\end{array}\right)
$$

after applying Gauss elimination, where $\theta=\left(\mu_{1} \lambda_{1}-\mu_{3} \lambda_{3}\right)-\left(\mu_{1} \lambda_{1}-\mu_{2} \lambda_{2}\right)\left(\frac{\lambda_{3}-\lambda_{1}}{\lambda_{2}-\lambda_{1}}\right)$. This matrix may lead to an ill-conditioned linear system when one of the diagonal 
entries blows up very fast or it becomes very close to zero. This situation occurs when the values of the function $\mu(\lambda)$ are getting very small or the difference between $\lambda_{2}$ and $\lambda_{1}$ is very close to zero.

2. The scale dependent, way in which $\mu_{2}$ has been chosen is not efficient. We need a scale independent (i.e a way depends on the physical properties of the system).

3. The rational approximation $\mu(\lambda) \approx \frac{\alpha \lambda+\beta}{\gamma \lambda+1}$ has a pole " $-\frac{1}{\gamma}$ " which is one of the advantages of rational function approximation. It gives better results when the approximated function has a pole. The pole of approximating the rational function may lead to a problem when the approximated function has no pole. In this situation, we want to reduce the function to a quadratic behavior.

\section{THE SAFEGUARDED TECHNIQUE}

A safeguarded interpolation method might include the following logic at each iteration. Using enough known information to find a new point without safeguards, the next step would evaluate the function value at the new point, and discard one of the old points to form a new point. However, a safeguarded procedure ensures that the new point is a "reasonable" point before evaluating the function at that point. The point is reasonable when it lies in the uncertainty interval (i.e it contains the solution) and it guarantees that the number of function evaluations required should not be significantly more than that required by the safeguard. Finally, safeguards are necessary to ensure that successive iterates are not "too close". It can happen that the new point is numerically indistinguishable from the previous best point, even when the new point is far from optimal. Even if the new point were accepted, no further progress would occur ${ }^{5}$. 


\subsection{The Best Choice of the Third Point}

In order to overcome the difficulties exposed in the previous section, we simplify the development by transforming the axes to pass through the point " $\lambda_{2}$ ", where $\lambda_{2} \in\left(\lambda_{1}, \lambda_{3}\right)$. The function $\mu(\lambda) \approx \frac{\alpha \lambda+\beta}{\gamma \lambda+1}$ can be interpolated at the points $\left(\lambda_{1}, \mu_{1}\right),\left(\lambda_{2}, \mu_{2}\right)$ and $\left(\lambda_{3}, \mu_{3}\right)$ to evaluate the coefficients $\alpha, \beta$ and $\gamma$. These values can be evaluated as follows :

$$
\begin{aligned}
& \beta=\mu_{2} \\
& \alpha=\frac{1}{\delta_{1}+\delta_{2}}\left[\left(\frac{\left(\delta_{1}+\delta_{2}\right) \mu_{2}-\left(\delta_{2} \mu_{1}+\delta_{1} \mu_{3}\right)}{\delta_{1} \delta_{2}\left(\mu_{3}-\mu_{1}\right)}\right)\left(\delta_{1} \mu_{1}+\delta_{2} \mu_{3}\right)+\left(\mu_{3}-\mu_{1}\right)\right] \\
& \gamma=\frac{\left(\delta_{1}+\delta_{2}\right) \mu_{2}-\left(\delta_{2} \mu_{1}+\delta_{1} \mu_{3}\right)}{\delta_{1} \delta_{2}\left(\mu_{3}-\mu_{1}\right)}
\end{aligned}
$$

where, $\delta_{1}=\lambda_{2}-\lambda_{1}, \delta_{2}=\lambda_{3}-\lambda_{2}$ and $\mu_{i}=\mu\left(\lambda_{i}\right), i=1,2,3$.

First, we satisfy the condition $\left(\delta_{1} \mu_{1}-1\right)\left(\delta_{2} \mu_{3}-1\right) \leq 0$ to ensure that the interval $\left[\delta_{1}, \delta_{2}\right]$ is the uncertainty interval. This can be done by increasing $\lambda_{3}$ and solve a linear eigenvalue problem to evaluate $\mu_{3}$. In the neighborhood of the solution, $\delta_{2}$ will be very small. This would cause the value of $\gamma$ to blow up. In this situation, we either use the safeguard or shrink the step. The value $\mu_{3}-\mu_{1}$ may lead to the same problem. In this case we employ the safeguard.

The choice of $\mu_{2}$ needs to be scale independent. We may interpolate linearly or use the bisection method (for one step only) between the two points $\left(\lambda_{1}, \mu_{1}\right)$ and $\left(\lambda_{3}, \mu_{3}\right)$ to evaluate $\lambda_{2}$. The solution of the linear eigenvalue problem, $\mathbf{A}\left(\lambda_{2}\right) \mathbf{x}=\mu\left(\lambda_{2}\right) \mathbf{x}$ gives $\mu_{2}=\max \mu\left(\lambda_{2}\right)$. This is expensive because it increases the number of function evaluations, each function value needs a solution of a large linear eigenvalue problem. The cheapest way to compute $\mu_{2}$ is to use the information in hand so that we do not need to solve a new linear eigenvalue problem. The line $\lambda=\lambda_{2}$ intersects the curve $\frac{1}{\lambda}$ in the point $\left(\lambda_{2}, \frac{1}{\lambda_{2}}\right)$ which gives the upper bound, $\frac{1}{\lambda_{2}}$ for the value $\mu_{2}$.

The function $\mu(\lambda)$ is monotone increasing on the uncertainty interval, (figure 1). Thus, the value $\mu_{2}$ has a lower bound $\mu_{1}$. The choice of $\mu_{2}$ must lie in the interval $\left[\mu_{1}, \frac{1}{\lambda_{2}}\right]$, figure 3. The function $\frac{\alpha \lambda+\beta}{\gamma \lambda+1}$ has a pole at " $\frac{-1}{\gamma}$ ". So, for some choices of $\mu_{2} \in\left[\mu_{1}, \frac{1}{\lambda_{2}}\right]$ the pole 
may lie inside the uncertainty interval, $\left[\lambda_{1}, \lambda_{3}\right]$. The best choice of $\mu_{2}$ is the value that lies in $\left[\mu_{1}, \frac{1}{\lambda_{2}}\right]$ and guarantees that the pole lies outside the uncertainty interval, see Appendix.

The behavior of the function $\mu(\lambda)$ and the shape of the rational approximation $\frac{\alpha \lambda+\beta}{\gamma \lambda+1}$ in figure 2 lead us to choose the left branch of this rational approximation to approximate our function. So, we are sure that the pole of this approximation should be away from the right end of the uncertainty interval $\left[\lambda_{1}, \lambda_{3}\right]$.

\subsection{Safeguarded Rational Zero-finding Technique}

This procedure is the modification of the rational zero-finder. It has many advantages:

1. The evaluation of $\mu_{2}$ in a scale independent way guarantees that the pole lies outside the uncetrainty interval. Moreover, we do not need to solve more linear eigenproblems.

2. Secant method is used as a safeguard when the values of the function $\mu(\lambda)$ is getting small, i.e the linear system is ill-conditioned.

3. This technique gives a much closer match to the actual curve of the function $\mu(\lambda)$. Also, it has the flexibility to search between $\lambda_{2}$ and $\lambda_{3}$ as well as between $\lambda_{1}$ and $\lambda_{2}$.

The following algorithm is a description for a complete iteration of this procedure. The required two points for the first iteration can be described as shown in subsection 3.1.

Algorithm 4.1 function $\left[\lambda_{1}, \lambda_{2}, \lambda_{3}\right]=$ saferational $\left(\lambda_{1}, \lambda_{2}, \lambda_{3}, \mu_{1}, \mu_{2}, \mu_{3}\right)$;

(1) Check Singularity :

If $\left(\mu_{2} \leq \epsilon_{1}\right)$ then,

$\left[\lambda_{2}\right] \leftarrow \operatorname{Secant}\left[\lambda_{1}, \lambda_{2}, \mu_{1}, \mu_{2}\right]$

return. 


\section{(2) Determine the third point :}

$$
\begin{aligned}
& \phi=\frac{\left(\mu_{2}-\mu_{1}\right)}{\left(\lambda_{2}-\lambda_{1}\right)} \\
& \Delta=\lambda_{2} \phi-\mu_{2} \\
& \lambda_{3} \leftarrow \lambda_{2}, \text { and } \mu_{3} \leftarrow \mu_{2} \\
& \eta=\left|\Delta^{2}+4 \phi\right| \\
& \lambda_{2}=\frac{(\Delta+\sqrt{\eta})}{2 \phi}, \delta_{1}=\lambda_{2}-\lambda_{1}, \delta_{2}=\lambda_{3}-\lambda_{2} \\
& \mu_{2}=0.5\left(\frac{1}{\lambda_{2}}+\mu_{1}\right) .
\end{aligned}
$$

(3) Solve :

$$
\left(\begin{array}{ccc}
-\delta_{1} & 1 & \delta_{1} \mu_{1} \\
0 & 1 & 0 \\
\delta_{2} & 1 & -\delta_{2} \mu_{3}
\end{array}\right)\left(\begin{array}{l}
\alpha \\
\beta \\
\gamma
\end{array}\right)=\left(\begin{array}{l}
\mu_{1} \\
\mu_{2} \\
\mu_{3}
\end{array}\right)
$$

For $\alpha, \beta$ and $\gamma$.

(4) Set : $\xi=\delta_{1} \delta_{2}\left(\mu_{3}-\mu_{1}\right)$

$\lambda_{1} \leftarrow \lambda_{2} ; \mu_{1} \leftarrow \mu_{2}$

(5) Solve : $\alpha \delta^{2}-(\gamma-\beta) \delta-1=0$, for the larger root;

(6) If $\left(\delta>\delta_{2}\right.$ and. $\left.\xi \leq \epsilon_{2}\right)$ then

$\lambda_{2}=\lambda_{1}+\epsilon_{2} \delta_{2}$

else if $\left(\delta>\delta_{2}\right.$.and. $\left.\xi>\epsilon_{2}\right)$ then

$\left[\lambda_{2}\right] \leftarrow \operatorname{Secant}\left(\lambda_{2}, \lambda_{3}, \mu_{2}, \mu_{3}\right)$

else

$\lambda_{2}=\lambda_{1}+\delta$

end if.

A new iteration starts, the matrix $\mathbf{A}(\lambda)$ is computed at the most updated value of $\lambda$ which is $\lambda_{2}$ (that comes from the zero-finder). A linear eigenvalue problem is solved to evaluate the value $\mu_{2}=\max \mu\left(\lambda_{2}\right)$ and the safeguarded rational zero-finder is called. This process is continued until the stopping criteria is satisfied and the first eigenvalue, $\lambda_{1}^{*}$ of 
the nonlinear eigenproblem is computed. Once the solution to the $j$-th nonlinear problem $\mu_{j}(\lambda)=\frac{1}{\lambda}$, under the condition $\|\mathbf{f}\|\left|\mathbf{e}_{k}^{t} \mathbf{y}_{j}\right|<\epsilon \mu_{j}$ (where $\mu_{j}=\max \mu\left(\lambda_{j}\right)$, the $j$-th largest generalized eigenvalue and its corresponding eigenvector, $\mathbf{y}_{\mathbf{j}}$ ) has been found. The $k$-step Lanczos factorization from the final iterative step of solving the $j$-th problem will be used to initiate the $(j+1)$-th problem. The solution to the $j$-th nonlinear problem, " $\lambda_{j}^{*}$ " provides an excellent initial guess to begin the zero-finding iteration for the next desired eigenvalue, $\lambda_{j+1}^{*}$. In this algorithm, we used $\epsilon_{1}=5 \times 10^{-2}$ and $\epsilon_{2}=10^{-5}$.

\section{THE NEW APPROACH}

The desired nonlinear eigenvalue problem (1.2) has a special structure and the goal of this technique is to compute a few of the smallest eigenvalues and their corresponding eigenvectors. The use of the mixed finite element model ${ }^{9}$ saves the computations because the matrix $\mathbf{K}$ is factored only once and this factorization is stored for all iterations. The new approach is based on the well known idea linearize, solve and then update. The eigensolver is the IRL method that has been determined to be well suited for solving the linear eigenproblems. The IRL technique has a close connection with the QR iteration and the ability to maintain an orthogonal basis of a predetermined size. The convergence of this technique is rapid for evaluating the largest few generalized eigenvalues, $\mu_{j}(\lambda)$ for specific values of the parameter $\lambda$. The safeguarded zero-finder is used to update the eigenvalue $\lambda$. This zero-finder is based on rational interpolation. The convergence rate of these types of zero-finders is very close to the convergence rate of the Newton-Raphson method, as reported in ${ }^{26}$. Moreover, there is no need for the derivative of the function.

The starting value $\lambda_{1}$ may be chosen to be zero at the beginning or it is already computed from the previous iteration. The starting vector $\mathbf{v}_{1}$ may be set to be $(1,1, \cdots, 1)^{t}$ or it can be generated by the rand function. The following algorithm introduces a complete iteration for evaluating the first smallest eigenvalue of problem (1.2).

\section{Algorithm 5.1}


function $\left[\lambda_{2}, \mathbf{V}\right]=\operatorname{safeLanc} z o s\left(\mathbf{K}, \mathbf{M}(\lambda), k, p\right.$, tol,$\left.\lambda_{\mathbf{l}}\right)$

(1) Factor : $\mathbf{K}$ into $\mathbf{L L}^{t}$

(2) Compute: $\mathbf{M}\left(\lambda_{1}\right)$;

3) $[\mathbf{T}, \mathbf{V}, \mathbf{f}] \leftarrow \operatorname{IRL}\left(\mathbf{M}\left(\lambda_{1}\right), \mathbf{L}, k, p, t o l\right)$;

(4) $\mu_{1} \leftarrow \mathbf{v}_{k+p}^{t} \mathrm{Tv}_{k+p}$,

$\lambda_{2} \leftarrow \frac{1}{\mu_{1}}$

(5) Compute: $\mathbf{M}\left(\lambda_{2}\right)$;

(6) $[\mathbf{T}, \mathbf{V}, \mathbf{f}] \leftarrow I R L\left(\mathbf{M}\left(\lambda_{\mathbf{2}}\right), \mathbf{L}, k, p, t o l\right)$

(7) $\mu_{2} \leftarrow \mathbf{v}_{k+p}^{t} \mathbf{T} \mathbf{v}_{k+p}$;

(8) Check the uncertainty of the interval

If $\left(\left(\lambda_{1} \mu_{1}-1\right)\left(\lambda_{2} \mu_{2}-1\right)>0\right)$ then

$\lambda_{2} \leftarrow \frac{3}{2} \lambda_{2}$ go to 5 ;

(9) Update $\lambda$

$\left[\lambda_{1}, \lambda_{2}, \lambda_{3}\right] \leftarrow$ saferational $\left(\lambda_{1}, \lambda_{2}, \lambda_{3}, \mu_{1}, \mu_{2}, \mu_{3}\right) ;$

(10) Check Convergence :

If, $\left|\lambda_{2}-\lambda_{1}\right| \leq 0.5 \epsilon\left(\lambda_{1}+\lambda_{2}\right)$, then stop;

(11) Compute: $\mathbf{M}\left(\lambda_{2}\right)$;

(12) $[\mathbf{T}, \mathbf{V}, \mathbf{f}] \leftarrow I R L\left(\mathbf{M}\left(\lambda_{\mathbf{2}}\right), \mathbf{L}, k, p, t o l\right)$;

$\mu_{2} \leftarrow \mathbf{v}_{k+p}^{t} \mathbf{T}_{\mathbf{v}_{k+p}}$

(13) $\lambda_{1} \leftarrow \lambda_{2}, \mu_{1} \leftarrow \mu_{2}$,

$\lambda_{2} \leftarrow \lambda_{3}, \mu_{2} \leftarrow \mu_{3} ;$

go to (9) 
This process continues until the stopping criterion is satisfied and the first smallest eigenvalue, $\lambda_{1}^{*}$ is computed. The second eigenvalue $\lambda_{2}^{*}$ can be computed by initializing the eigen-solver and the zero-finder using the previous information. The initial vector $\mathbf{v}_{\mathbf{1}}$ for the IRL will be the first column of the matrix $\mathrm{V}$ from the last $\mathrm{k}$-step factorization from the previous iteration. We solve the linear eigenvalue problem

$$
\mathbf{A}\left(\lambda_{2}\right) \mathbf{x}=\mu\left(\lambda_{2}\right) \mathbf{x}
$$

where, $\lambda_{2}=\frac{1}{\mu_{2}\left(\lambda_{1}\right)}$ and $\mu_{2}\left(\lambda_{1}\right)$ is the second largest generalized eigenvalue of the last linear eigenvalue problem has been solved for finding $\lambda_{1}^{*}$. The two points that the zero-finder needs are set to be $\left(\lambda_{1}, \mu_{1}\right)=\left(\lambda_{1}^{*}, \mu_{2}\left(\lambda_{1}\right)\right)$ and $\left(\lambda_{2}, \mu_{2}\right)=\left(\frac{1}{\mu_{2}\left(\lambda_{1}\right)}, \mu_{2}\left(\lambda_{2}\right)\right)$, where $\mu_{2}\left(\lambda_{2}\right)$ is the second largest generalized eigenvalue of problem (5.1), see figure 1 .

This strategy takes at least solution of two linear eigenvalue problems to compute the first nonlinear eigenvalue, $\lambda_{1}^{*}$. It also takes at least solving one linear eigenvalue problem to find each one from the rest, $\lambda_{j}^{*}, j=2,3, \cdots, 10$.

\section{COMPUTATIONAL RESULTS}

Efficient and portable Fortran software of these algorithms is achieved by relying on the LAPACK and BLAS subroutine libraries. This new technique has been implemented and tested on Sparc Station1 machine, under release 4.1.1 of Sun Unix. The cost of operations were clearly dominated by the matrix vector products and the linear solvers routines. The solution of the nonlinear eigenvalue problem is solutions to a sequence of linear eigenvalue subproblems. So, parallelism may be invoked through the Level 2 BLAS and also through the matrix vector product routines ${ }^{24}$.

In practice, the stopping criterion is very sensitive to the problem in hand, so a special care should be taken in its choice. The two common stopping conditions for the nonlinear equations may be stated as follows :

$$
\left|f\left(\lambda_{j+1}\right)\right| \leq \epsilon \text { and } \frac{\left|\lambda_{\mathrm{j}+1}-\lambda_{\mathrm{j}}\right|}{\max \left(\left|\lambda_{\mathrm{j}+1}\right|,\left|\lambda_{\mathrm{j}}\right|\right)} \leq \epsilon
$$


where, $\lambda_{j+1}$ is the latest approximated value of $\lambda, \lambda_{j}$ is the previous one and $\epsilon=(\text { macheps })^{\frac{1}{2}}$, where macheps is a very small positive quantity attainable on the computer ${ }^{6}$. The stopping criterion $\left|\lambda_{j+1}-\lambda_{j}\right| \leq \epsilon$ has the disadvantage of being machine dependent. The second inequality in (6.1) is scale independent when $\lambda \neq 0$. This inequality is a reasonable stopping criterion for the zero-finding techniques, but it will be stringent when $\lambda_{j+1}, \lambda_{j}$ are very small $^{11}$. We used the stopping criterion

$$
\left|\lambda_{j+1}-\lambda_{j}\right| \leq \frac{\epsilon}{2}\left|\lambda_{j+1}+\lambda_{j}\right|, \text { where } \epsilon=10^{-6}
$$

to stop the outer iteration (the zero-finder). The inner iteration (the eigen-solver) stops when $\left|\mathbf{e}_{k}^{t} \mathbf{y}_{j}\right|\|\mathbf{f}\| \leq \epsilon_{3} \mu_{j}$, where $\mathbf{y}_{j}$ is the $j-$ th Ritz vector corresponding to the Ritz value approximating the required spectrum, $\mu_{j}$ is the generalized eigenvalue for the problem $\mathbf{A}\left(\lambda_{\mathbf{j}}\right) \mathbf{x}=\mu\left(\lambda_{\mathbf{j}}\right) \mathbf{x}$ and $\epsilon_{3}=10^{-9}$ is in double precision arithmetic. Stopping this $\mathrm{k}$-step Lanczos iteration produces a good initial guess for computing the next eigenvalue $\lambda_{j+1}^{*}$.

\subsection{Comparison with Some Existing Techniques}

The smallest ten eigenvalues and their corresponding eigenvectors of the test problems are obtained using the safeguarded Lanczos with rational interpolation method developed here, the Lanczos-Based technique with linear interpolation that was introduced in ${ }^{23}$ and the determinant search approach that was presented $\mathrm{in}^{29}$. The purpose of solving these eigensystems is to illustrate the computational efficiency of the new approach for large scale nonlinear structural eigensystems and to compare this approach with both the LanczosBased procedure and the multiple determinant search technique.

The multiple determinant parabolic interpolation method has been developed by Williams and Kennedy ${ }^{29}$ for solving the dependent eigenvalue problem (1.1). This method is based on factorize the dynamic stiffness matrix $[\mathbf{K}(\lambda)-\lambda \mathbf{M}(\lambda)]$, evaluate the determinant of this matrix and then update $\lambda$. The use of the combined zero-finding (the quadratic interpolation with bisection method) offers computational advantage over the bisection technique that has been used in a traditional determinant search $\operatorname{method}^{30}$, 
especially for higher accuracy requirements. Results from ${ }^{29}$ show a $10 \%$ improvement in computational measurements over Simpson's method ${ }^{18}$. Unfortunately, this technique is very expensive for large systems because of evaluating the determinant of the dynamic stiffness matrix. This evaluation requires factorization of the entire matrix at each step of the search. In addition, the parameterized determinant curves have a potential erratic behavior $^{29}$. behavior [29].

The Lanczos-Based eigensolution technique evaluates a subset of the natural frequencies and modes from the dependent eigenproblems in structural dynamics. These systems are associated with the mixed finite element modeling procedure which is concerned with the polynomial and frequency dependent displacement fields in formulating the system matrices ${ }^{23}$. This approach is based upon the IRL method for solving the linear eigenvalue subproblems and the secant method for updating the eigenvalue $\lambda$. The Lanczos-Based procedure gave remarkable results but it took 4 function evaluations for each eigenvalue ${ }^{23}$. eigenvalue [23]. In addition, the linear interpolation is a special case of the rational approximation that we used to approximate the generalized eigenvalue curves $\mu_{j}(\lambda)$. On the other hand, the new technique approximates these parameterized curves by rational functions and it has the ability to update the parameter $\lambda$ twice in each step. Moreover, the independent way of choosing the third point controls the location of the pole and helps to minimize the number of function evaluations.

The result,s obtained from solving a few test problems with different dimensions are listed in tables 1 through 3. As shown, the safeguarded Lanczos technique gives a better improvement over Lanczos-Based and determinant search techniques in minimizing the number of function evaluations, saving computational time and giving a small function value at the solution. It is clear that the three methods are competitive for the small size problems, but for the large size ones the safeguarded Lanczos method seems to perform best. In particular, for problems approaching $10^{3}$ degrees-of-freedom the number of function evaluations by safeguarded Lanczos method is approximately one-half of the number of function evaluations by Lanczos-Based technique. It is also approximately $\frac{1}{20}$ of the number 
of function evaluations by the determinant search procedure. Each function evaluation takes solution of one linear eigenvalue problem (in Lanczos techniques), but it takes one factorization of the dynamic stiffness matrix (in the multiple determinant search approach).

Figures 5 and 6 demonstrate the total time required to find the smallest ten eigenvalues as a function of the problem dimension. Figure 5 shows that for problems with dimension less than 100 degrees-of-freedom the Lanczos-Based technique with secant interpolation and the multiple determinant parabolic interpolation approach are competitive with the safeguarded Lanczos nonlinear eigenvalue method using rational interpolation. However, as the problem dimension increases, the safeguarded Lanczos method becomes more efficient as shown in figure 6 .

\subsection{Numerical Experiments}

The test problems are a frame-type structures involving different unconstrained degreesof-freedom (sizes between 24 and $10^{3}$ ) using the mixed finite element model that contains a frequency independent stiffness matrix, $\mathbf{K}$ and a frequency dependent mass matrix $\mathbf{M}(\lambda)$. The mixed model formulation has advantages over the computationally intensive exact finite element model. In the later model the mass and stiffness matrices are dependent on the parameter $\lambda$. Any approach for solving it requires more computational operations for refactorization and redecomposition of the dynamic stiffness matrix at each step. In addition, the mixed finite element model dominates the conventional finite element model which involves discretization error ${ }^{23}$.

The linear eigen-solver that we used (IRL) only requires matrix vector products. It has many other advantages such as maintenance of orthogonality, fixed storage and rapid convergence rate for evaluating a subset of the extremes eigenvalues and their corresponding eigenvectors.

The rational approximations have the advantage over polynomial approximations of any order. They are used to approximate any function with or without a pole. Moreover, they are considered as the generalization of the linear interpolation. The secant method 
that is based on linear interpolation used to update the eigenvalue $\lambda$ in the Lanczos-Based technique. In contrast, our zero-finder approach that is based on rational function interpolation is used to approximate the parameterized curves $\mu_{j}(\lambda)$ and updates the parameter $\lambda$. The value of $\mu_{2}$ was computed to be dependent on the physical properties of the system and control the pole.

In all the results reported in this contribution, we have used the exact shifts, the values $\mathrm{k}=10, \mathrm{p}=20$ in algorithm 5.1 , the condition $\left|\lambda_{j+1}-\lambda_{j}\right| \leq 10^{-6}$ to terminate the multiple determinant search iteration and the inequality (6.2) to terminate the iterations of LanczosBased and safeguarded Lanczos approaches. The natural frequencies $\omega$ are computed by using the formula $\omega=\frac{\sqrt{\lambda}}{2 \pi}$ and their corresponding modes are the eigenvectors.

In the tables 1 through 3, the safeguarded Lanczos method computes the first eigenvalue by solving at least two linear eigenvalue subproblems because we do not have any information. The computation of each eigenvalue from the rest needs at least solution of one linear eigenproblem. If the generalized eigenvalues $\mu(\lambda)$ exceed $5 \times 10^{-2}$ the step of the safeguarded rational zero-finder is equivalent to one step from secant method (for finding the third point for the rational interpolation) and one step from rational zero-finder for updating the parameter $\lambda$. These features have been reflected in the computations. For example, the evaluation of the first eigenvalue takes one function evaluation less than secant method (see tables 1 through 3).

\section{Notations :}

$\mathrm{Sr}$ No $=$ the eigenvalues number, $\lambda=$ the value of the nonlinear eigenvalue.

$\mathrm{t} / \mathrm{s}=$ the time in seconds for evaluating the eigenvalue,

$\operatorname{err}=\left|\lambda_{j+1}-\lambda_{j}\right|$

$\mathrm{fe}=$ the number of function evaluations for computing each eigenvalue. 


\begin{tabular}{|c|c|c|c|c|c|c|c|c|c|c|c|c|}
\hline \multirow{2}{*}{$\begin{array}{l}\mathrm{Sr} \\
\text { No } \\
\end{array}$} & \multicolumn{4}{|c|}{ Safeguarded with Lanczos } & \multicolumn{4}{|c|}{ Secant with Lanczos } & \multicolumn{4}{|c|}{ Determinant search method } \\
\hline & $\lambda$ & fe & $\mathrm{t} / \mathrm{s}$ & err & $\lambda$ & fe & $\mathrm{t} / \mathrm{s}$ & err & $\lambda$ & fe & $\mathrm{t} / \mathrm{s}$ & err \\
\hline 1 & 0.037 & 2 & 7.92 & $1 \mathrm{D}-11$ & 0.037 & 3 & 12.0 & $5 \mathrm{D}-11$ & 0.037 & 35 & 470 . & $7 \mathrm{D}-7$ \\
\hline 2 & 0.348 & 1 & 4.19 & 1D-9 & 0.348 & 2 & 8.5 & 2D-9 & 0.348 & 21 & 282. & $5 \mathrm{D}-7$ \\
\hline 3 & 1.005 & 1 & 4.85 & $8 D-9$ & 1.005 & 2 & 9.8 & $2 \mathrm{D}-8$ & 1.005 & 20 & 268. & $9 \mathrm{D}-7$ \\
\hline 4 & 2.073 & 1 & 6.11 & $2 \mathrm{D}-8$ & 2.073 & 2 & 11.8 & 7D-8 & 2.073 & 21 & 283 & $9 \mathrm{D}-7$ \\
\hline 5 & 3.629 & 1 & 5.70 & $6 \mathrm{D}-8$ & 3.629 & 2 & 11.3 & $1 \mathrm{D}-7$ & 3.629 & 21 & 283 & $9 \mathrm{D}-7$ \\
\hline 6 & 5.731 & 1 & 6.73 & $1 \mathrm{D}-7$ & 5.731 & 2 & 13.3 & $4 \mathrm{D}-7$ & 5.731 & 19 & 255 & $9 \mathrm{D}-7$ \\
\hline 7 & 8.368 & 2 & 11.8 & 1D-7 & 8.368 & 2 & 11.98 & 1D-6 & 8.368 & 21 & 280 & $9 \mathrm{D}-7$ \\
\hline 8 & 11.38 & 1 & 6.59 & 1D-7 & 11.38 & 2 & 13.3 & $8 D-7$ & 11.38 & 20 & 267 & $5 \mathrm{D}-7$ \\
\hline 9 & 14.39 & 1 & 7.22 & 1D-7 & 14.39 & 2 & 14.5 & 2D-7 & 14.39 & 21 & 282 & $9 \mathrm{D}-7$ \\
\hline 10 & 16.71 & 1 & 7.40 & $6 \mathrm{D}-8$ & 16.71 & 2 & 14.6 & 1D-7 & 16.71 & 19 & 255 & $8 D-7$ \\
\hline
\end{tabular}

Table 1: The smallest 10 eigenvalues for a system of size 330 degrees-of-freedom, the half bandwidth of the stiffness matrix $=55$ and the number of constraints $=33$. In these two tables 2 and 3, the determinant search technique takes a large number of the dynamic stiffness matrix factorization. On the other hand Lanczos techniques take a few number of linear eigenvalue subproblems solutions and give more accuracy. As shown, Safeguarded Lanczos method minimizes the number of function evaluations.

\begin{tabular}{|c|c|c|c|c|c|c|c|c|c|c|c|c|}
\hline Sr & \multicolumn{3}{|c|}{ Safeguarded with Lanczos } & \multicolumn{3}{|c|}{ Secant with Lanczos } & \multicolumn{3}{|c|}{ Determinant search method } \\
\cline { 2 - 12 } No & $\lambda$ & fe & t/s & err & $\lambda$ & fe & t/s & err & $\lambda$ & fe & t/s & err \\
\hline 1 & 0.009 & 2 & 32.5 & 1D-12 & 0.009 & 3 & 49. & $1 \mathrm{D}-13$ & 0.010 & 36 & 6058. & $8 \mathrm{D}-7$ \\
2 & 0.085 & 1 & 18.1 & $8 \mathrm{D}-11$ & 0.085 & 2 & 36.9 & $4 \mathrm{D}-12$ & 0.085 & 20 & 3361. & $9 \mathrm{D}-7$ \\
3 & 0.239 & 1 & 21.4 & $4 \mathrm{D}-10$ & 0.239 & 2 & 43.1 & $1 \mathrm{D}-11$ & 0.239 & 21 & 3526. & $7 \mathrm{D}-7$ \\
4 & 0.477 & 1 & 22.4 & $1 \mathrm{D}-9$ & 0.477 & 2 & 45.1 & $8 \mathrm{D}-11$ & 0.477 & 21 & 3524. & $9 \mathrm{D}-7$ \\
5 & 0.805 & 1 & 29.8 & $3 \mathrm{D}-9$ & 0.805 & 2 & 59.6 & $3 \mathrm{D}-10$ & 0.805 & 21 & 3525. & $9 \mathrm{D}-7$ \\
6 & 1.232 & 1 & 29.8 & $6 \mathrm{D}-9$ & 1.232 & 2 & 59.2 & $1 \mathrm{D}-9$ & 1.232 & 19 & 3212. & $8 \mathrm{D}-7$ \\
7 & 1.769 & 1 & 32.2 & 1D-8 & 1.769 & 2 & 64.9 & $5 \mathrm{D}-9$ & 1.769 & 21 & 3526. & $9 \mathrm{D}-7$ \\
8 & 2.425 & 1 & 36.3 & $1 \mathrm{D}-8$ & 2.425 & 2 & 73.3 & $1 \mathrm{D}-8$ & 2.425 & 20 & 3358. & $5 \mathrm{D}-7$ \\
9 & 3.211 & 1 & 39.2 & $2 \mathrm{D}-8$ & 3.211 & 2 & 79.1 & $3 \mathrm{D}-8$ & 3.211 & 21 & 3526. & $9 \mathrm{D}-7$ \\
10 & 4.136 & 1 & 39.2 & $4 \mathrm{D}-8$ & 4.136 & 2 & 79.1 & $6 \mathrm{D}-8$ & 4.136 & 19 & 3191. & $9 \mathrm{D}-7$ \\
\hline
\end{tabular}

Table 2: The smallest 10 eigenvalues for a system of size 960 degrees of freedom, the half bandwidth of the stiffness matrix $=80$ and the number of constraints $=48$. 


\begin{tabular}{|c|c|c|c|c|c|c|c|c|c|c|c|c|}
\hline Sr & \multicolumn{3}{|c|}{ Safeguarded with Lanczos } & \multicolumn{3}{c|}{ Secant with Lanczos } & \multicolumn{3}{|c|}{ Determinant search method } \\
\cline { 2 - 11 } No & $\lambda$ & fe & t/s & err & $\lambda$ & fe & t/s & err & $\lambda$ & fe & t/s & err \\
\hline 1 & 0.244 & 2 & 0.67 & $7 \mathrm{D}-10$ & 0.244 & 3 & 0.9 & $1 \mathrm{D}-8$ & 0.244 & 20 & 5.93 & $2 \mathrm{D}-7$ \\
2 & 2.413 & 2 & 0.58 & $5 \mathrm{D}-8$ & 2.413 & 2 & 0.6 & $1 \mathrm{D}-7$ & 2.413 & 6 & 1.77 & $5 \mathrm{D}-8$ \\
3 & 7.514 & 2 & 0.82 & $2 \mathrm{D}-7$ & 7.514 & 2 & 0.7 & $3 \mathrm{D}-6$ & 7.514 & 8 & 2.34 & $5 \mathrm{D}-8$ \\
4 & 14.92 & 2 & 1.08 & $3 \mathrm{D}-7$ & 14.92 & 2 & 1.1 & $4 \mathrm{D}-6$ & 14.92 & 10 & 2.90 & $4 \mathrm{D}-9$ \\
5 & 52.29 & 4 & 2.44 & $1 \mathrm{D}-5$ & 52.29 & 4 & 2.5 & $5 \mathrm{D}-5$ & 52.29 & 12 & 3.56 & $4 \mathrm{D}-11$ \\
6 & 60.37 & 3 & 2.18 & $4 \mathrm{D}-5$ & 60.37 & 4 & 3.0 & $3 \mathrm{D}-5$ & 60.37 & 14 & 4.09 & $3 \mathrm{D}-7$ \\
7 & 68.91 & 4 & 3.25 & $5 \mathrm{D}-5$ & 68.91 & 4 & 3.2 & $1 \mathrm{D}-5$ & 68.91 & 7 & 2.06 & $2 \mathrm{D}-12$ \\
8 & 79.80 & 4 & 3.73 & 9D-6 & 79.80 & 4 & 3.8 & $1 \mathrm{D}-5$ & 79.80 & 7 & 2.05 & $6 \mathrm{D}-8$ \\
9 & 81.65 & 3 & 3.13 & 1D-5 & 81.65 & 3 & 3.0 & $1 \mathrm{D}-5$ & 81.65 & 7 & 2.03 & $6 \mathrm{D}-11$ \\
10 & 95.29 & 4 & 4.11 & 7D-6 & 95.29 & 4 & 4.1 & $1 \mathrm{D}-6$ & 95.29 & 9 & 2.63 & $2 \mathrm{D}-13$ \\
\hline
\end{tabular}

Table 3: The smallest ten eigenvalues for a system of size 60 degrees-of-freedom, the half bandwidth of the stiffness matrix $=25$ and the number of constraints $=15$. The safeguarded zero-finder computes the first four eigenvalues without using the safeguard and the safeguard is used for computing the rest because of the values of $\mu(\lambda)$. Determinant search method gives more accuracy over the Lanczos techniques but it takes several number of the dynamic stiffness matrix factorization.

\section{Concluding Remarks}

In this paper, we have established a numerical algorithm for solving a class of nonlinear eigenproblems. The eigen-solver (IRL) has been reformulated to be well-suited to solve the linear eigenvalue subproblems that arise. For updating the parameter $\lambda$, we have developed a zero-finding technique which is based on the rational interpolation. This type of interpolation can be used to approximate any function of any degree, even when this function has a pole. The third point in this technique has been chosen in a scale independent way. This would guarantee that the pole lies away from the right end of the uncertainty interval. The safeguard has been used to ensure that the successive points are not too close.

The new solution technique is used for approximating frequency-dependent, nonlinear eigensystems associated with exact free vibration analysis of frame structure. This technique is used in affiliation with the mixed finite element formulation presented in ${ }^{9}$. This formulation employs both the frequency-dependent and polynomial fields in generating the mass and stiffness matrices, respectively. Our algorithm is capable of producing accurate 
results for large scale problems. Furthermore, we have illustrated its effectiveness in this context through numerical experiments.

The new methodology of the solution developed here is designed to compute a certain set of parameterized nonlinear curves at given values of the parameter " $\lambda$ " through the eigen-solver and update this parameter through the safeguarded zero-finder procedure. The IRL approach is a variant of the highly successful implicit $\mathbf{Q R}$ technique for small dense problems with the Lanczos method for very large symmetric sparse systems. Our method shows that the use of the new eigensolution procedure accurately approximates the exact eigenvalues and the eigenvectors of the nonlinear eigensystems associated with exact vibration analysis. This was illustrated by evaluating the solution for test problems that include the generated problems in $^{23}$. We also compare the results of the new approach with the results of known published techniques ${ }^{23,29}$. Moreover, our approach proves to be competitive in terms of computational efficiency not only for large scale problems but also for small problem sizes.

\section{Acknowledgements}

I gratefully acknowledge Prof. Danny C. Sorensen my graduate advisor for reading the manuscript, making some useful comments and corrections, and for providing the ARPACK library which is written by him and P. A. Vu. I would also like to acknowledge H.A.Smith and R.K.Singh for providing me an experimental code for generating the mass and stiffness matrices and for their discussions that lead to establish a stronger version of the test problems than the one used in my preliminary version of this work.

This work was supported in part by NSF cooperative agreement CCR-9120008 and by the Department of Energy contract DE-FG0F-91ER25103. 


\section{Appendix}

Any value of the function $\mu(\lambda)$ is a generalized eigenvalue of the symmetric positive definite matrix, $A(\lambda)$ at a specific value of the parameter $\lambda$ and it is monotone increasing, figure 1 . Since, $\mu(\lambda) \approx \frac{\alpha \delta+\beta}{\gamma \delta+1}$, where $\delta=\lambda-\lambda_{2}$ is always positive quantity then

$$
\begin{gathered}
\alpha \delta+\beta>0 \text { and } \gamma \delta+1>0, \text { or } \\
\alpha \delta+\beta<0 \text { and } \gamma \delta+1<0 .
\end{gathered}
$$

From inequalities $(7.1)$, we have $\delta>\frac{-1}{\gamma}$. We also know that $\delta$ is always positive so that $\gamma>0$ or $\gamma<0$. From inequalities (7.2), we get $\delta<\frac{-1}{\gamma}$ which is true when $\gamma<0$. From this discussion we have the following important remarks.

1. The pole lies away from the right end of the interval of uncertainty, $\left[\delta_{1}, \delta_{2}\right]$, when $\delta_{2}<\frac{-1}{\gamma}$ and $\gamma<0$.

2. If $-\delta_{1}>\frac{-1}{\gamma}$ and $\gamma>0$ then the pole lies away from the left end of the uncertainty interval, $\left[\delta_{1}, \delta_{2}\right]$.

The following lemmas determine conditions on the third point, $\mu_{2}$. These conditions guarantee that the pole lies outside the uncertainty interval.

Lemma 7.1 The pole of the rational function $\mu(\lambda) \approx \frac{\alpha \delta+\beta}{\gamma \delta+1}$ lies away from the right end of the interval $\left[\delta_{1}, \delta_{2}\right]$ if and only if

$$
\mu_{2}>\mu_{1} \text { and } \gamma<0
$$

Proof: Suppose that the pole lies away from the right end of the interval $\left[\delta_{1}, \delta_{2}\right]$ " $\delta_{2}<\frac{-1}{\gamma}$ and $\gamma<0$ " then, $\gamma \delta_{2}+1>0$. But $\mu_{3} \approx \frac{\alpha \delta_{2}+\beta}{\gamma \delta_{2}+1}$ is always positive. Thus,

$$
\alpha \delta_{2}+\beta>0
$$

Introducing equations (4.1) and (4.2) in the inequality (7.3) and rearranging terms give

$$
\mu_{2}\left[\frac{\delta_{2}\left(\delta_{1} \mu_{1}+\delta_{2} \mu_{3}\right)}{\delta_{1} \delta_{2}\left(\mu_{3}-\mu_{1}\right)}+1\right]>\frac{\delta_{2}\left(\delta_{2} \mu_{1}+\delta_{1} \mu_{3}\right)\left(\delta_{1} \mu_{1}+\delta_{2} \mu_{3}\right)}{\left(\delta_{1}+\delta_{2}\right) \delta_{1} \delta_{2}\left(\mu_{3}-\mu_{1}\right)}-\frac{\delta_{2}\left(\mu_{3}-\mu_{1}\right)}{\delta_{1}+\delta_{2}} .
$$


Since $\delta_{1} \delta_{2}\left(\mu_{3}-\mu_{1}\right)>0$, we get

$\mu_{2}\left[\delta_{2}\left(\delta_{1} \mu_{1}+\delta_{2} \mu_{3}\right)+\delta_{1} \delta_{2}\left(\mu_{3}-\mu_{1}\right)\right]>\frac{\delta_{2}}{\delta_{1}+\delta_{2}}\left[\left(\delta_{2} \mu_{1}+\delta_{1} \mu_{3}\right)\left(\delta_{1} \mu_{1}+\delta_{2} \mu_{3}\right)-\delta_{1} \delta_{2}\left(\mu_{3}-\mu_{1}\right)^{2}\right]$.

Eliminating the similar terms and using the assumption $\gamma<0$, we have

$$
\mu_{2}>\mu_{1} \text { and } \gamma<0
$$

For proving the other direction of the lemma, suppose that condition (7.4) is satisfied. Using (4.1) and (4.2) and rearranging terms give

$$
\alpha \delta_{2}+\beta>\frac{\delta_{2}\left(\delta_{1}+\delta_{2}\right) \mu_{1} \mu_{3}}{\delta_{1} \delta_{2}\left(\mu_{3}-\mu_{1}\right)}-\delta_{2} \frac{\left(\delta_{2} \mu_{1}+\delta_{1} \mu_{3}\right)\left(\delta_{1} \mu_{1}+\delta_{2} \mu_{3}\right)}{\left(\delta_{1}+\delta_{2}\right) \delta_{1} \delta_{2}\left(\mu_{3}-\mu_{1}\right)}+\delta_{2} \frac{\mu_{3}-\mu_{1}}{\delta_{1}+\delta_{2}} .
$$

The right hand side of this inequality is zero. So, $\alpha \delta_{2}+\beta>0$ but $\mu\left(\delta_{2}\right)>0$ then $\gamma \delta_{2}>-1$. Since $\delta_{2}$ is positive, hence $\gamma>0$ or $\gamma<0$. The inequalities $\delta_{2}<-\frac{1}{\gamma}$ and $\gamma<0$ assure that the pole lies away from the right end of the uncertainty interval $\left[\delta_{1}, \delta_{2}\right]$. The other possibility $\left(\delta_{2}>-\frac{1}{\gamma}\right.$ and $\left.\gamma>0\right)$ is rejected because the pole may lie inside the interval $\left[\delta_{1}, \delta_{2}\right]$.

Lemma 7.2 The pole of the rutional function $\mu(\lambda) \approx \frac{\alpha \delta+\beta}{\gamma \delta+1}$ lies to the left end of the interval $\left[\delta_{1}, \delta_{2}\right]$ if and only if

$$
\mu_{2}>\mu_{3} \text { and } \gamma>0
$$

Proof: Suppose that the pole lies away from the left end of the interval $\left[\delta_{1}, \delta_{2}\right]$. So, $-\delta_{1}>\frac{-1}{\gamma}$ and $\gamma>0$. Since $\mu_{1} \approx \frac{-\alpha \delta_{1}+\beta}{-\gamma \delta_{1}+1}$ is positive and $-\gamma \delta_{1}+1>0$, then $-\alpha \delta_{1}+\beta>0$. Using inequalities (4.1) and (4.2) and rearranging the terms give

$$
-\alpha \delta_{1}+\beta=-\delta_{1}\left[\frac{\mu_{2}\left(\delta_{1} \mu_{1}+\delta_{2} \mu_{3}\right)}{\delta_{1} \delta_{2}\left(\mu_{3}-\mu_{1}\right)}-\frac{\left(\delta_{2} \mu_{1}+\delta_{1} \mu_{3}\right)\left(\delta_{1} \mu_{1}+\delta_{2} \mu_{3}\right)}{\left(\delta_{1}+\delta_{2}\right) \delta_{1} \delta_{2}\left(\mu_{3}-\mu_{1}\right)}+\frac{\mu_{3}-\mu_{1}}{\delta_{1}+\delta_{2}}\right]+\mu_{2}
$$

But, $-\alpha \delta_{1}+\beta$ and $\delta_{1} \delta_{2}\left(\mu_{3}-\mu_{1}\right)$ are positive, then

$$
\mu_{2}\left[-\delta_{1}\left(\delta_{1} \mu_{1}+\delta_{2} \mu_{3}\right)+\delta_{1} \delta_{2}\left(\mu_{3}-\mu_{1}\right)\right]>\frac{-\delta_{1}}{\delta_{1}+\delta_{2}}\left[\left(\delta_{2} \mu_{1}+\delta_{1} \mu_{3}\right)\left(\delta_{1} \mu_{1}+\delta_{2} \mu_{3}\right)-\delta_{1} \delta_{2}\left(\mu_{3}-\mu_{1}\right)^{2}\right] .
$$

Eliminating the similar tems and using the assumption $\gamma>0$ give

$$
\mu_{2}>\mu_{3} \text { and } \gamma>0
$$


The proof of the second direction of the lemma can be derived by assuming the condition (7.5). Using formulas (4.1) and (4.2) and rearranging terms give

$$
-\alpha \delta_{1}+\beta>\frac{-\delta_{1}\left(\delta_{1}+\delta_{2}\right) \mu_{1} \mu_{3}}{\delta_{1} \delta_{2}\left(\mu_{3}-\mu_{1}\right)}+\delta_{1} \frac{\left(\delta_{2} \mu_{1}+\delta_{1} \mu_{3}\right)\left(\delta_{1} \mu_{1}+\delta_{2} \mu_{3}\right)}{\left(\delta_{1}+\delta_{2}\right) \delta_{1} \delta_{2}\left(\mu_{3}-\mu_{1}\right)}-\delta_{1} \frac{\mu_{3}-\mu_{1}}{\delta_{1}+\delta_{2}}
$$

The right hand side of this inequality equals zero, then $-\alpha \delta_{1}+\beta>0$ but $\mu_{1}$ is positive. So, we deduce that $-\delta_{1}>\frac{-1}{\gamma}$ with $\gamma>0$ which completes the proof.

\section{References}

[1] W.E. Arnoldi, "The principle of minimized iterations in the solution of the matrix eigenvalue problem," Quart. Appl. Math.,9, 17-29,(1951) .

[2] J. Cullum and R. A. Wiloughby, Algorithms for Large Symmetric Eigenvalue Computations, Birkhauser Boston, Inc, 1985.

[3] G. H. Golub and C. F. Van Loan, Matrix Computations, The Johns Hopkins University Press, 1983.

[4] W. L. Hallauer and R. Y. L. Liu, "Beam bending-torsion dynamic stiffness method for calculation of exact vibration modes," Journal of Sound and Vibration, 85, 105-113, (1982).

[5] J. C. Bus and T. J. Dekker, "Two efficient algorithms with guaranteed convergence for finding a zero of a function," ACM Trans. Math. Software, 1, 330-345, (1975).

[6] J. E. Dennis JR. and R. B. Schnabel, "Numerical Methods for Unconstrained Optimization and Nonlinear Equations," Prentice-Hall, Englewood Cliffs, New Jersey, 1983.

[7] C. Lanczos, "An iteration method for the solution of the eigenvalue problem of the linear differential and integral operators," Res. Nat. Bur. Standards, 45, 255-282, (1950). 
[8] M. R. Abdel-Aziz, Numerical Safeguarded Use of the Implicit Restarted Lanczos Algorithm for Solving Nonlinear Eigenvalue Problems and its Monoyonicity Analysis, $\mathrm{PhD}$. Thesis of Computational and Applied Mathematics Department, Rice University, 1993.

[9] R. J. Melosh and H. A. Smith, "New formulation for vibration analysis," Journal of Engineering Mechanics, 115, 543-554, (1989).

[10] J. J. More' and D. C. Sorensen, "Computing a Trust Region Step," SIAM J. sci. stat. comput., 14, No.3, (1983).

[11] J. R. Bunch, C. P. Nielsen and D. C. Sorensen, "Rank-One Modification of the Symmetric Eigenproblem," Numer. Math., 31, 31-48, (1978).

[12] B. N. Parlett, "The Symmetric Eigenvalue Problem," Prentice-Hall, Englewood Cliffs, NJ, 1980.

[13] J. S. Prezmieniecki, "Theory of Matrix Structural Analysis," McGraw-Hill Book Company, New York, 1968.

[14] T. H. Richards and Y. T. Leung, "An accurate method in structural vibration analysis," Journal of Sound and Vibration, 55, 363-367, (1977).

[15] A. Ruhe, "Algorithms for the Nonlinear Eigenvalue Problem," SIAM J. Numer. Anal., 10, 674-689, (1973).

[16] A. Ruhe, "Rational Krylov sequence methods for eigenvalue computation," Linear Algebra App., 58, 391-405, (1984).

[17] Y. Saad, "Large Scale Nonsymmetric Eigenvalue Problems," John Wiley and Sons, New York, 1992.

[18] A. Simpson, "On the solution of $s(w) x=0$ by a Newtonian procedures," Journal of sound and vibration, 97, 153-164, (1978). 
[19] A. Simpson and B. Tabarrok, “On Kron's Eigenvalue Procedure and Related Methods of Frequency Analysis," Quarterly Journal of Mechanics and Applied Mathematics, Pt. I, 1-36, (1967).

[20] M. R. Abdel-Aziz, "A Monotonicity Analysis Theory for the Dependent Eigenvalues of the Vibrating Systems in Structural Dynamics," (submitted to the Journal of engineering Mathematics).

[21] H. A. Smith and R. J. Melosh, "Unsymmetric formulation for vibration analysis of frames," Computers and Structures, 36, 531-537, (1990).

[22] D. C. Sorensen, "Implicit Application of Polynomial Filters in a k-step Arnoldi Method," SIAM Journal on matrix analysis, 13, 357-385, (1991).

[23] H. A. Smith, D. C. Sorensen and R. K. Singh, "A Lanczos-Based Eigensolution Technique For Exact Vibration Analysis of Skeletal Structures," International Journal for Numerical Methods in Engineering, To appear, (1993).

[24] J. J. Dongarra, I. S. Duff, D. C. Sorensen and H. A. Van der Vorst, "Solving Linear Systems on Vector and Shared Memory Computers," SIAM, Philadelphia, 1991.

[25] G. W. Stewart, "Introduction to Matrix Computations," Academic Press, New York, 1973.

[26] J. Stoer and R. Bulirsch, "Introduction to Numerical Analysis," Springer-Verlag, New York, Heidelberg, Berlin, 1983.

[27] P. Swannell, "Automatic computation of the natural frequencies of structural frames using an exact matrix technique," Theory and Practice in Finite Element Structural Analysis, University of Tokyo Press, 289-303, (1973).

[28] J. H. Wilkinson, "The Algebraic Eigenvalue Problems," Oxford, England, Claredon Press, 1965. 
[29] F. W. Williams and D. Kennedy, "Reliable use of determinants to solve nonlinear structural eigenvalue problems efficiently," International Journal for Numerical Methods in Engineering, 26, 1825-1841, (1988).

[30] F. W. Williams and W. H. Wittrick, "An automatic computational procedure for calculating natural frequencies of skeletal structures," International Journal of Mechanical Science, 12, 781-791, (1970).

[31] W. H. Wittrick and F. W. Williams, "A general algorithm for computing natural frequencies of elastic structures," Quarterly Journal of Mechanics and Applied Mathematics, 24, 263-284, (1971).

[32] I. M. Yang, "TECHNICAL NOTE: An Iterative Method for Solving Nonlinear Eigenvalue Problems in Matrix Form," Computers and Structures, 29, 353-354, (1988). 


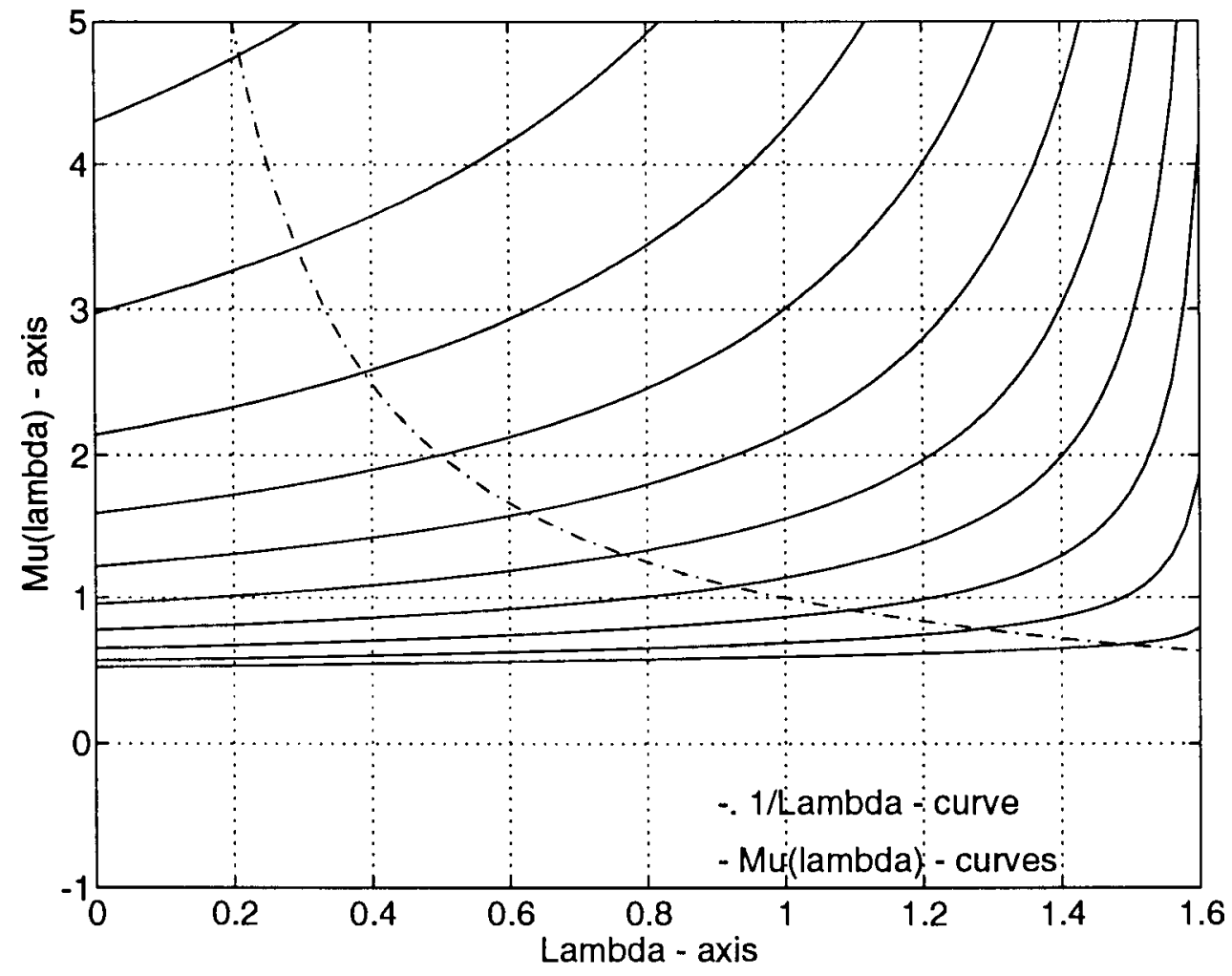

Figure 1: graphically depicts the smallest ten eigenvalues for the nonlinear eigenproblem (1.2) as the intersection points between the $\mu$-curves and the $\frac{1}{\lambda}$-curve. It is also illustrates the behavior of the spectrum of the matrix $\mathbf{L}^{-1} \mathbf{M}(\lambda) \mathbf{L}^{-t}$, where $\mathbf{K}=\mathbf{L} \mathbf{L}^{t}$. These global matrices $K$ and $M(\lambda)$ are generated by using the assembly process for the elemental stiffness and mass matrices that are associated with the mixed finite element model, for the axial vibration. This process may be explained as follows : for element $i$ with nodes $i$ and $i+1$, the two rows and columns of the elemental matrix are added into rows and columns $i$ and $i+1$ of the matrix for the complete system. After the final stage of the assembly process, we eliminate the first row and the first column from the global matrix due to the boundary conditions. 


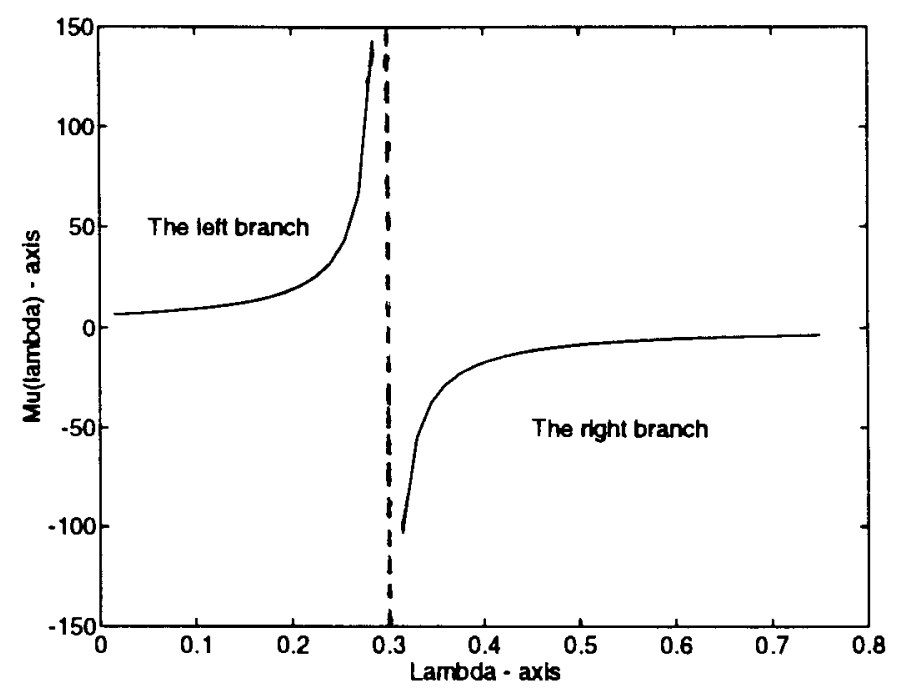

Figure 2: An example for a rational function

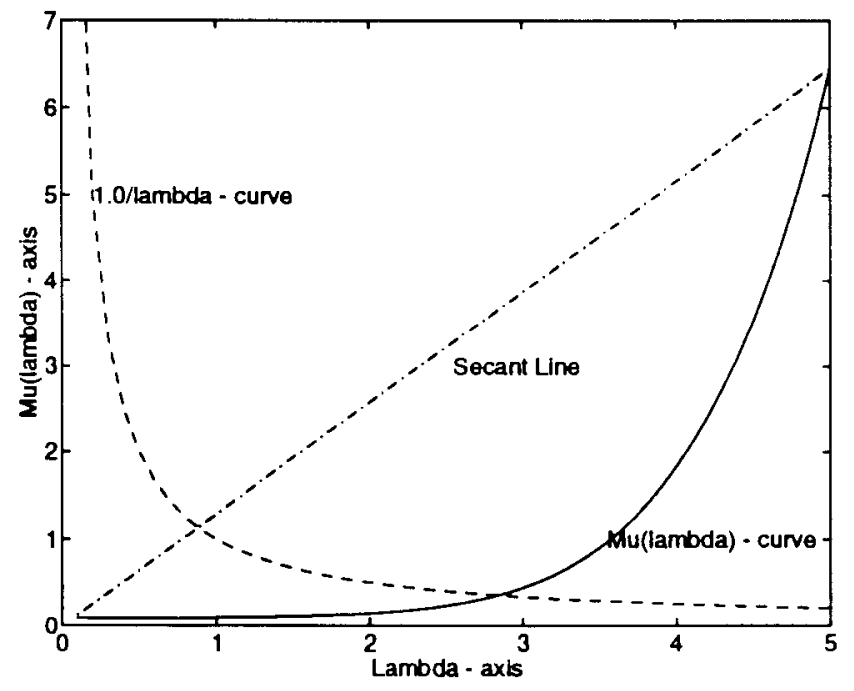

Figure 3: gives the upper and lower bounds of $\mu_{2}$. The intersection points of the secant line with $\mu(\lambda)$ and $\frac{1}{\lambda}$ curves are: $\left(\lambda_{1}, \mu_{1}\right),\left(\lambda_{2}, \frac{1}{\lambda_{2}}\right)$ and $\left(\lambda_{3}, \mu_{3}\right)$ from left to right. 


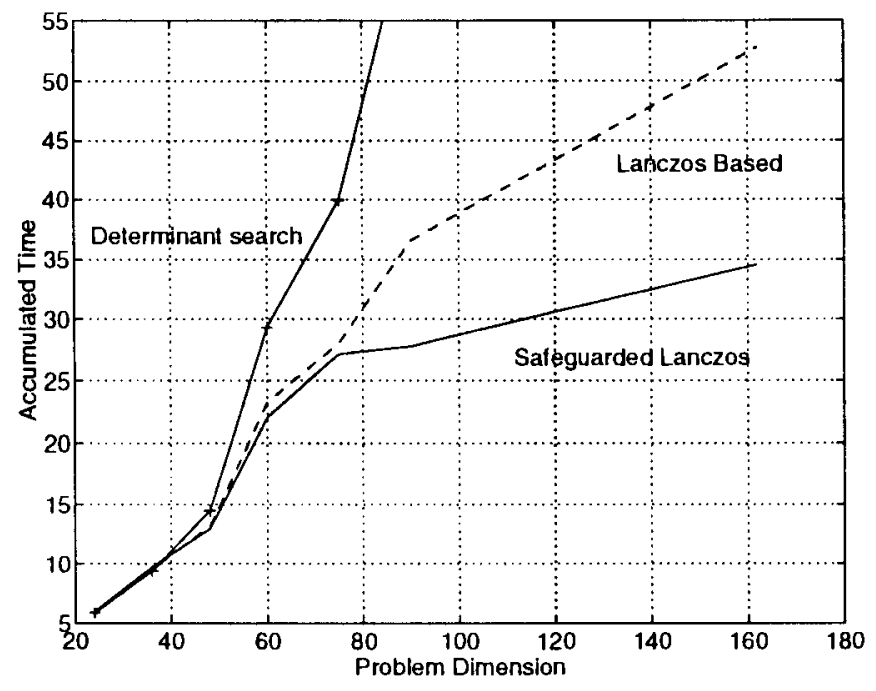

Figure 4: The accumulated time for small problems

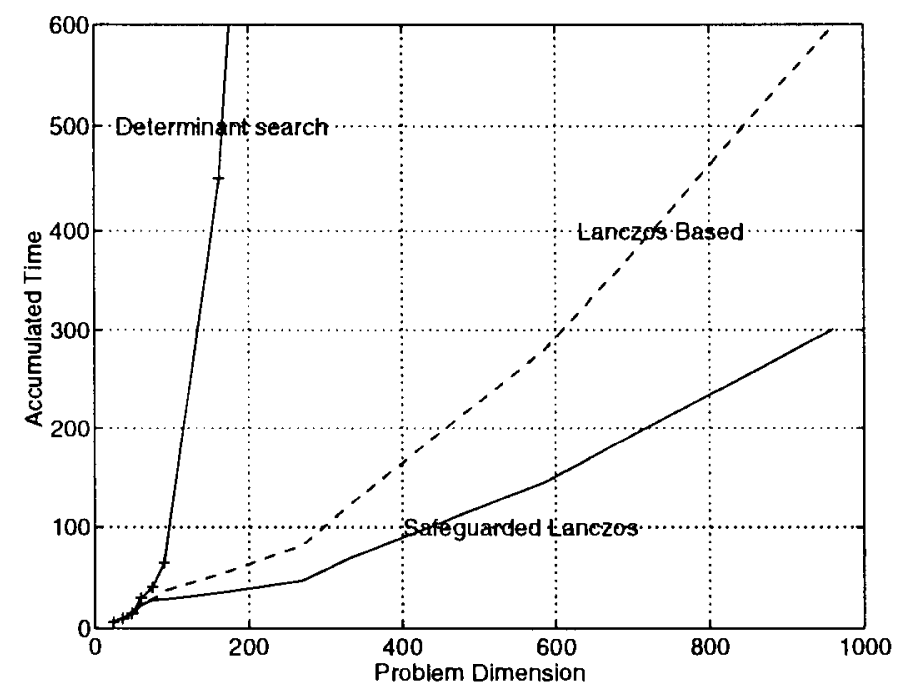

Figure 5: The accumulated time for large problems 